\title{
Foldable Conductive Cellulose Fiber Networks Modified by Graphene Nanoplatelet-Bio-Based Composites
}

\author{
Pietro Cataldi, Ilker S. Bayer,* Francesco Bonaccorso, Vittorio Pellegrini, \\ Athanassia Athanassiou, and Roberto Cingolani*
}

Truly foldable flexible electronic components require a foldable substrate modified with a conducting material that can retain its electrical conductivity and mechanical integrity even after hard mechanical manipulations and multiple folding events. Here, such a material exploiting the combination of all-biodegradable components (substrate and the polymer matrix) and graphene nanoplatelets is designed and fabricated. A commercially available thermoplastic starch-based polymer (Mater-Bi) and graphene nanoplatelets are simultaneously dispersed in an organic solvent to formulate conductive inks. The inks are spray painted on pure cellulose sheets and hot-pressed into their fiber network after drying. The resultant nanostructured flexible composites display excellent isotropic electrical conductivity, reaching very low sheet resistance value $\approx 10 \Omega \mathrm{sq}^{-1}$, depending on the relative concentration between the biopolymer and the graphene nanoplatelets. Transmission electron microscopy results indicated that during hot-pressing, graphene nanoplatelets are physically embedded into the cellulose fibers, resulting in high electrical conductivity of the flexible composite. The paper-like flexible conductors can withstand many severe folding events, maintaining their mechanical and electrical properties and showing only a slight decrease of their electrical conductivity with respect to the unfolded counterparts. Unlike conductive paper technologies, the proposed paper-like flexible conductors demonstrate both sides isotropic conductivity due to pressure-induced impregnation.

repeated folding and unfolding events. ${ }^{[2]}$ One of the most popular foldable substrates is paper. ${ }^{[3]}$ It has already been implemented as an alternative to plastic as a lightweight substrate for low-cost, flexible, and versatile roll-to-roll printed electronics, including transistors, solar cells, and batteries. ${ }^{[4,5]}$ The printing paper is sized, i.e., it contains various additives such as calcium carbonate and starch as fillers to tune color, gloss, printability, and water resistance. ${ }^{[6-8]}$ A side effect of sizing is generally linked with the closure of the pores of the paper, preventing the full and effective impregnation of conductive inks. As a result, in the vast majority of printing papers, the conductive paths are onesided only, thus not possessing isotropic electrical conductivity. ${ }^{[9]}$ To overcome this issue, here we have used sheets made of pure cellulose fibers as foldable substrates.

Graphene is a 2D conducting material consisting of carbon atoms arranged in a hexagonal lattice. ${ }^{[10]}$ It is currently being studied extensively for electronic applications due to its high electrical conductivity, optical transparency, gas barrier property, robustness, flexibility, and envi-

\section{Introduction}

Flexible electronic components are generally associated with bendable substrates. ${ }^{[1]}$ However, truly foldable electronics require preservation of electrical conductivity under severe deformation or multiple folding events. It is quite challenging to design and fabricate such materials, since in addition to the substrates, also the deposited conductors should be foldable, maintaining continuous electrical conductive paths after

P. Cataldi, Dr. I. S. Bayer, Dr. A. Athanassiou, Prof. R. Cingolani

Smart Materials, Istituto Italiano di Tecnologia Via Morego 30, 16163 Genova, Italy

E-mail: ilker.bayer@iit.it; roberto.cingolani@iit.it

Dr. F. Bonaccorso, Dr. V. Pellegrini, Prof. R. Cingolani

Graphene Laboratories

Istituto Italiano di Tecnologia

Via Morego 30, 16163 Genova, Italy

DOI: 10.1002/aelm.201500224 ronmental stability. ${ }^{[11-14]}$ One big challenge in the preparation of graphene/polymer composites is to maintain well-dispersed graphene flakes in the polymer matrices. ${ }^{[15]}$ However, apart from truly monolayer graphene also sheet-like or flake-like graphitic materials, already available for scale-up production, are frequently used for the development of polymer composites. ${ }^{[16]}$ For this reason, new graphitic materials are being continuously designed with significant variations in layer number, lateral dimension, rotational faulting, and chemical modification, suitable for compounding into soft polymeric or inorganic materials exploitable in a large variety of applications. ${ }^{[17,18]}$

Here, we present a protocol to create a foldable conductive paper-like material by impregnating a composite of starch-based biopolymer and graphene nanoplatelets (GnPs) into meltblown nonwoven cellulose fiber fleece substrates. ${ }^{[19]}$ The process is initiated by spraying the biopolymer-GnPs ink onto the cellulose substrates followed by hot-pressing it into the fibrous texture. After the impregnation process, a highly isotropic bendable conductor is obtained. The conductors display remarkable folding resistance under repeated folding-unfolding events, 
preserving their original electrical conductivity, i.e., only a decrease of $29 \%$ is observed. We present detailed electrical characteristics including percolation properties as a function of $\mathrm{GnP}$ concentration and lateral flake size, as well as folding resistance by monitoring the increase in electrical resistance. Finally, we demonstrate the operation of light-emitting diodes (LEDs) powered through the developed composite material as an electrical conductor. We demonstrate the LEDs operation both before and after deformation by severe folding events of the composite.

\section{Results and Discussion}

In order to develop the flexible conductors, fibrous cellulose substrates were sprayed on both sides with the polymeric conductive ink that was subsequently hot-pressed at $175{ }^{\circ} \mathrm{C}$ and 320 bars for $1 / 2 \mathrm{~h}$ into the fibrous structure. For the hot-pressing process, a bench-top automatic hydraulic hot press was used. The as-prepared conductors were sandwiched between two Teflon foils in order to prevent adhesion to the heated metal surfaces during polymer melting. The conductive inks to be sprayed were prepared by dispersing various amounts of GnPs in biopolymeric dispersions. Chloroform was used as the common solvent since it is volatile, having a boiling point of $61.3{ }^{\circ} \mathrm{C}$ with a low vapor pressure $\left(21.28 \mathrm{kPa}\right.$ at $\left.20{ }^{\circ} \mathrm{C}\right)$. As biodegradable polymer we used Mater-Bi, a commercial and well-studied thermoplastic starch-aliphatic polyester blend fabricated by melt extrusion. ${ }^{[2,21]}$ Two different commercially available GnPs were selected as conductive additives and they were named as SGnPs (Strem Chemicals) and DGnPs (Directa Plus). Additionally, colloidal graphite flakes ${ }^{[22]}$ were also used for comparison purposes (see Experimental Section for details).

Size and thickness characteristics of SGnP and DGnP were measured by atomic force microscopy (AFM) and dynamic light scattering (DLS) analysis. Results concerning SGnPs are presented here for brevity. For the dispersions used for DLS measurements (Figure S6, Supporting Information), we have used the same experimental conditions, but without the addition of the biopolymer, exploited for the preparation of the composites. The dispersions were prepared by dispersing $\approx 0.5 \%$ by weight of SGnP in chloroform in order to avoid light scattering effect during measurements. It must be mentioned however, that in dilute dispersions and in the absence of the biopolymer, the cavitation effect, ${ }^{[23]}$ i.e., the creation and subsequent collapse of bubbles or voids in liquids due to pressure fluctuations that induce the exfoliation and in-plane fracture of the GnPs during ultrasonication, ${ }^{[24]}$ is more effective, with respect to the $\mathrm{GnP}$ dispersions containing the biopolymer. However, such measurements can still provide a useful indication on the size distribution range of the GnPs. The size distributions data of the SGnPs are presented in Figure S6 (Supporting Information) indicating an average lateral size of $\approx 2 \mu \mathrm{m}$. This is smaller than the value reported by the manufacturer $(\approx 5 \mu \mathrm{m})$. Such discrepancy could be linked to the sonication process, ${ }^{[12,24]}$ which tends to reduce both lateral size and thickness of the flakes. ${ }^{[12,24]} \mathrm{AFM}$ measurements indicated that the thickness of SGnPs is $\approx 3 \mathrm{~nm}$, corresponding to more than 10 graphene layers. The electrical conductivity of pure SGnP and DGnP films prepared by spray
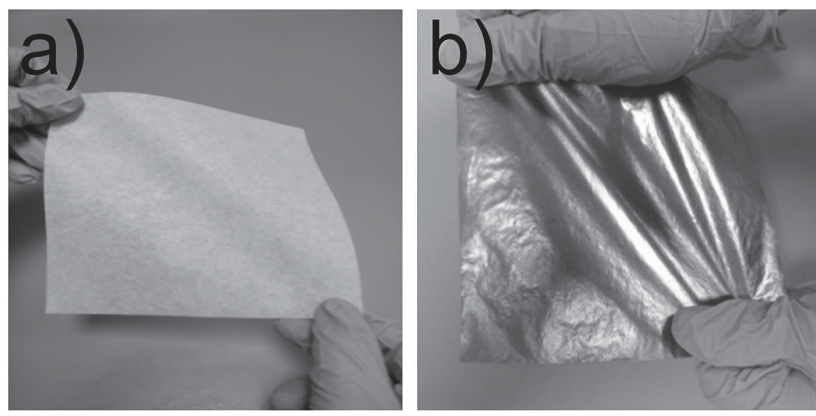

Figure 1. a) Photograph of the porous cellulose fiber substrate and b) photograph of the paper-like conductor after impregnating with a Mater-Bi-SGnPs (1:1).

coating on the cellulose substrates was measured following the same procedure carried out for the composites. Both SGnPsand DGnPs-based films demonstrated an average sheet resistance $\left(R_{\mathrm{s}}\right)$ value of $6 \Omega \mathrm{sq}^{-1}$ lower than the one shown by the composites, i.e., $\approx 10 \Omega \mathrm{sq}^{-1}$. Raman analysis (Figure S1, Supporting Information) of the starting materials with Mater-Bi demonstrates that the dispersion process and the GnPs/MaterBi composite fabrication do not induce additional defects with respect to the starting materials. Transmission electron microscopy (TEM) analysis of the GnPs indicates that both samples have lateral sizes in the micrometer range. However, as shown in the TEM images (Figure S2, Supporting Information), the lateral size of SGnP agglomerates was about $3 \mu \mathrm{m}$ while DGnP agglomerates display larger lateral size with dimension of about $15 \mu \mathrm{m}$. According to the manufacturer, the size of the colloidal graphite flakes is $10 \mu \mathrm{m}$.

Figure 1 shows photographs of the meltblown nonwoven cellulose substrate (30 $\mathrm{mm}$ thick) before and after spraying both sides with the biopolymer-GnP composite and subsequently hot-pressing it for impregnation. Note that the impregnation process can be adapted to any polymer fiber network as long as the network contains open micropores. In fact, the process is similar to paper sizing since it is used to fill cellulosic pores with specific agents in order to impart mechanical and water absorption resistance and gloss to paper. Figure S3 (Supporting Information) compares scanning electron microscope (SEM) images of pure cellulose substrate (Figure S3a, Supporting Information) and a standard-sized Xerox printing paper (Figure S3b, Supporting Information). the SEM analysis clearly shows that the pores of the printing paper are closed due to sizing effect. ${ }^{[7]}$

In order to achieve electrical conduction throughout these large area flexible substrates with low isotropic ohmic resistance, the conductive composites needed to exceed specific GnP concentrations, rto achieve the percolation of the GnPs within the biopolymer matrix. Indeed, as shown in the sheet resistance graphs obtained by the modified fibrous substrates at different loadings of $\mathrm{GnP}$ and colloidal graphite (Figure 2a), the composites maintain insulating properties (hundreds of $\mathrm{G} \Omega \mathrm{sq}^{-1}$ ) until approximately $6 \mathrm{wt} \% \mathrm{GnP}$ concentration. These results are in agreement with the behavior of various other polymergraphene composites reported in literature. ${ }^{[25]}$ Below this threshold, the current-voltage $(I-V)$ curves display highly hysteretic non-ohmic behavior (see the top right inset in Figure 2). 

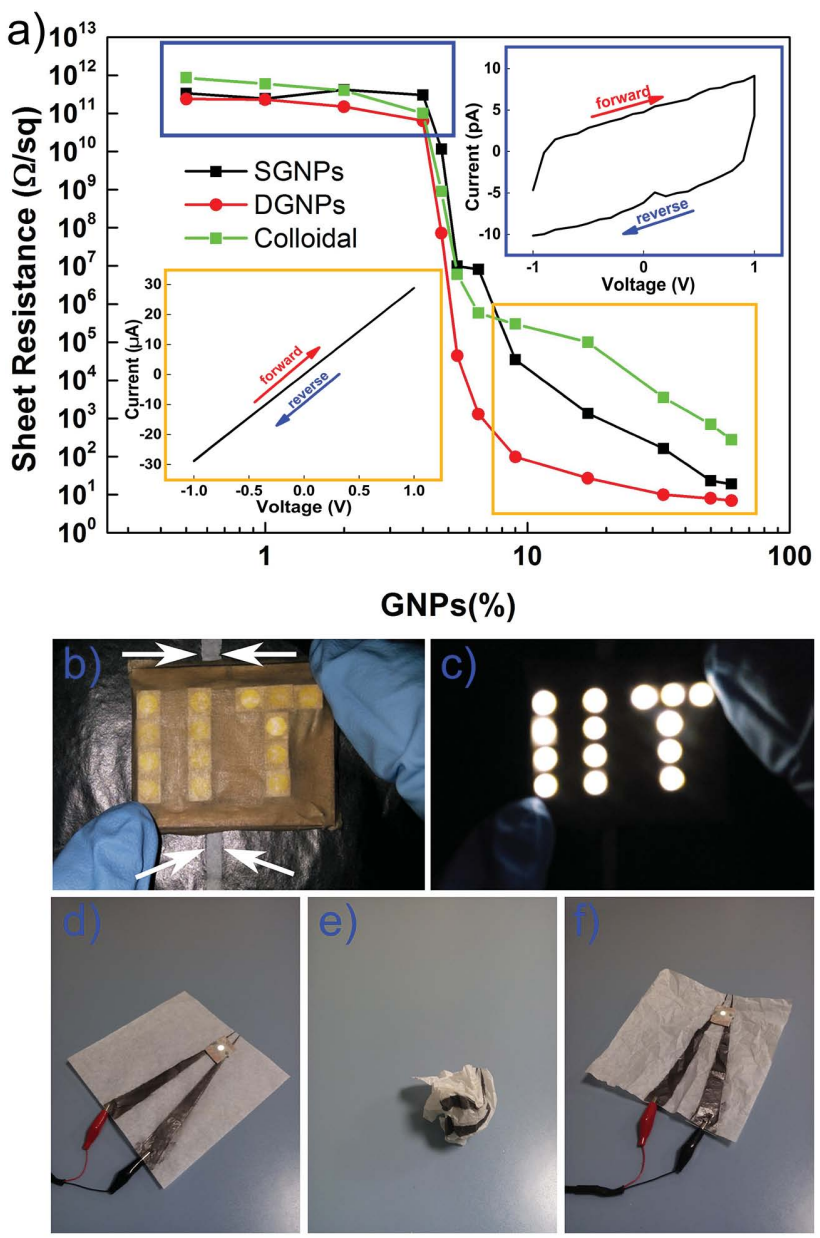

Figure 2. a) Four-probe sheet resistance measurements, $R_{\mathrm{s}}$, as a function of $\mathrm{GnP}$ and colloidal graphite concentration. The blue inset corresponds to the $I-V$ measurements taken before percolation took place while the yellow (ohmic) to the one after percolation. b) Photograph (taken in the dark) of a LED chip placed on the foldable conductor. The arrows indicate a line left uncoated and unimpregnated in order to avoid short circuiting. c) Once the foldable paper-like conductor is connected to a 5 V USB port of a computer the chip is lit up. This photograph was also taken in dark. d) Photograph of a similar concept as in (b) for a single LED attached to a conducting base with conducting paths embedded into the cellulose sheet. Note that alligator clips can easily grasp the foldable conductors causing no damage indicating mechanical robustness of the paper-like conductors. e) Photograph of the paper-like conductor squashed and pressed into a wrinkled ball by hands. f) Photograph of the paper-like conductor after undoing and flattening the squashed ball in (d). The LED light still works after this severe mechanical treatment.

It is important to note that electrical measurements reported herein are for one-sided samples (ink only sprayed on one face), however, hot pressing of both surface sprayed samples produced identical results due to excellent isotropy as a result of impregnation.

Above this threshold instead, it was found that GnPs with larger lateral size (DGnPs) can reduce the sheet resistance of the flexible conductors to $\approx 10 \Omega \mathrm{sq}^{-1}$ with much less concentration compared to SGnPs. In particular, as seen in Figure 2a, in order to fabricate a paper-like conductor with very low sheet resistance (10 $\Omega \mathrm{sq}^{-1}$ ), $20 \mathrm{wt} \% \mathrm{DGnPs}$ need to be compounded with Mater-Bi and incorporated into the fibrous network, compared to $50 \mathrm{wt} \% \mathrm{SGnPs}$. This result indicates how the average lateral sizes of the GnPs are as important as the degree of their dispersion in the polymer matrix. ${ }^{[26]}$ Moreover, the use of few layer graphene platelets is indeed justified if one compares the sheet resistance values with colloidal graphite with a loading of $20 \mathrm{wt} \%$. Resistance values achieved by colloidal graphite are at least two orders of magnitude higher than GnPs. Once percolation threshold was reached, the $I-V$ curves of all the paper-like conductors have shown ohmic behavior with hysteresis-free current-voltage behavior, as illustrated in the lower left inset of Figure 2a.

Examples of excellent electrical conductivity and ability to work after squashing these paper-like conductors loaded with SGnPs are shown in Figure 2b-f. In Figure 2b, photograph of a chip carrying 14 LED lights is shown in contact with the conductor. In the middle of the conductor, there is an intentionally made line of discontinuity (non-impregnated region) in order to avoid short circuiting. The circuit is powered by a $5 \mathrm{~V}$ USB cable and the LEDs are lit up as shown in Figure 2c. The chip can freely slide over the conductor surface while being always lit up. Similarly, it is possible to form lines or other patterns on the cellulose substrate, as shown in Figure 2d, by first spray coating through a mask and subsequently hot pressing. In this case, a single LED light is lit up. This sample was later on squashed into a ball as seen in Figure 2e. After undoing the wrinkled ball and flattening it, the LED chip was found to work normally as seen in Figure $2 \mathrm{f}$. Such a high mechanical resistance to severe folding damage while maintaining good electrical conductivity is extremely important for a large variety of applications such as flexible and wearable electronics ${ }^{[14]}$ with any kind of fibrous network. ${ }^{[27]}$

In order to better analyze the percolation threshold of the GnPs within the composites, we present the results based on conductivity $(\sigma)$ versus volume fraction $(\Phi)$ as shown in Figure 3a. ${ }^{[28]}$ Note that the percolation analysis was identical for one-side and two-side impregnated composites; hence only results from one side impregnated samples are reported here. The percolation theory concerning well-defined spherical particles indicates that conductivity $\sigma$ is proportional to the volume fraction $\Phi$ :

$\sigma \propto\left(\Phi-\Phi_{\mathrm{c}}\right)^{\tau}$

where $\Phi_{\mathrm{c}}$ is the percolation threshold (volume fraction at which the composite conducts) and $\tau$ is the universal critical exponent. ${ }^{[29,30]}$ This exponent can indicate the type of percolation. In fact, if the system creates a continuum network of contacts between conductive micro-/nano-objects, $\tau$ assumes the value $\approx 1.3$, while for a $2 \mathrm{D}$ conductive system $\tau$ would be $\approx 1.0$ and, finally, for a $3 \mathrm{D}$ conductive network $\tau$ would be $\approx 2.0 . .^{[31,32]}$ For graphene flakes or nanoplates, this theory can have limitations. ${ }^{[3]}$ However, as a first-order approximation, it is still being used in the literature. ${ }^{[34-36]}$ The critical exponent can have higher values due to different phenomena happening at the micro-/nanoscale such as a high number of tunneling events, contact resistance, and the presence of structural imperfections in between the conductive media (Swiss cheese model). ${ }^{[32,37]}$ In order to extract the $\tau$ values from the measurements, $\log \sigma$ 


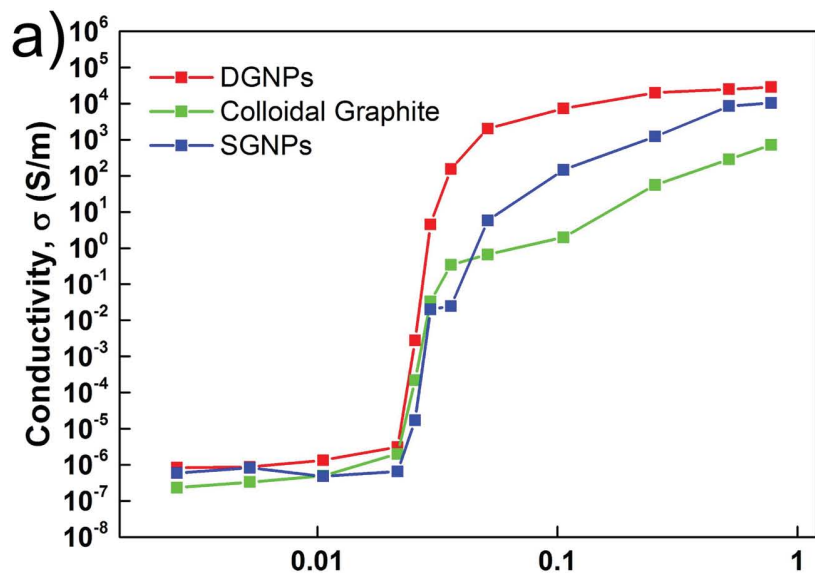

Filler volume fraction, $\Phi$

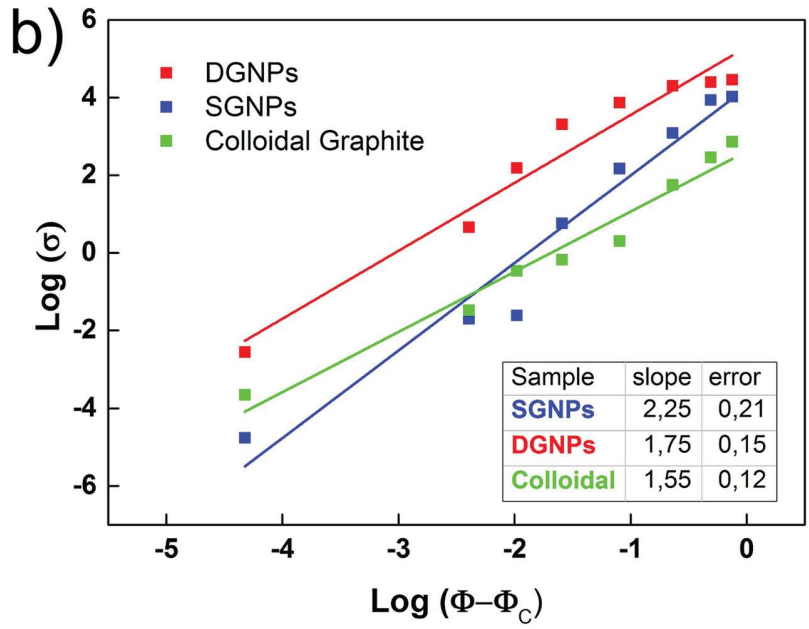

Figure 3. Percolation analysis of the system. a) Conductivity $\sigma$ as a function of GnP volume fraction $\Phi$, b) Linear $\log \sigma$ versus $\log \left(\Phi-\Phi_{c}\right)$ plot where $\Phi_{c}$ is the percolation threshold, which was found to be 0.0255 . The inset table shows calculated slope corresponding to the parameter $\tau$.

versus $\log \left(\Phi-\Phi_{\mathrm{c}}\right)$ was plotted in Figure $4 \mathrm{~b}$. A linear fit to the data allows extraction of $\tau$ as the slope (see inset to Figure 3b). Interestingly, for both DGnPs and SGnPs, $\tau$ was calculated to be close to 2 indicating a 3D percolation mechanism in the form of direct continuum contact even though the GnPs are 2D fillers. ${ }^{[32,37]}$ Also, colloidal graphite was found to percolate by direct contact, but the calculated $\tau$ value of 1.3 indicates a conducting mechanism closer to that of a $2 \mathrm{D}$ conducting composite system. ${ }^{[32]}$

As aforementioned, spraying both sides of the cellulose fiber sheets and the subsequent hot pressing process produced truly isotropic conductors in which there were no differences in conductivities $I-V$ characteristics between both surfaces as well as through the sample bulk. This is due to the fact that hot pressing of one-side sprayed samples causes the other face of the cellulose fiber sheets to be conductive due to the impregnation process, as shown in Figure 4a. In fact, this isotropy in conductivity is linked to the structure of the chosen cellulose substrate (meltblown cellulose) that does not contain any sizing agents, which are instead common to Xerox paper. As shown
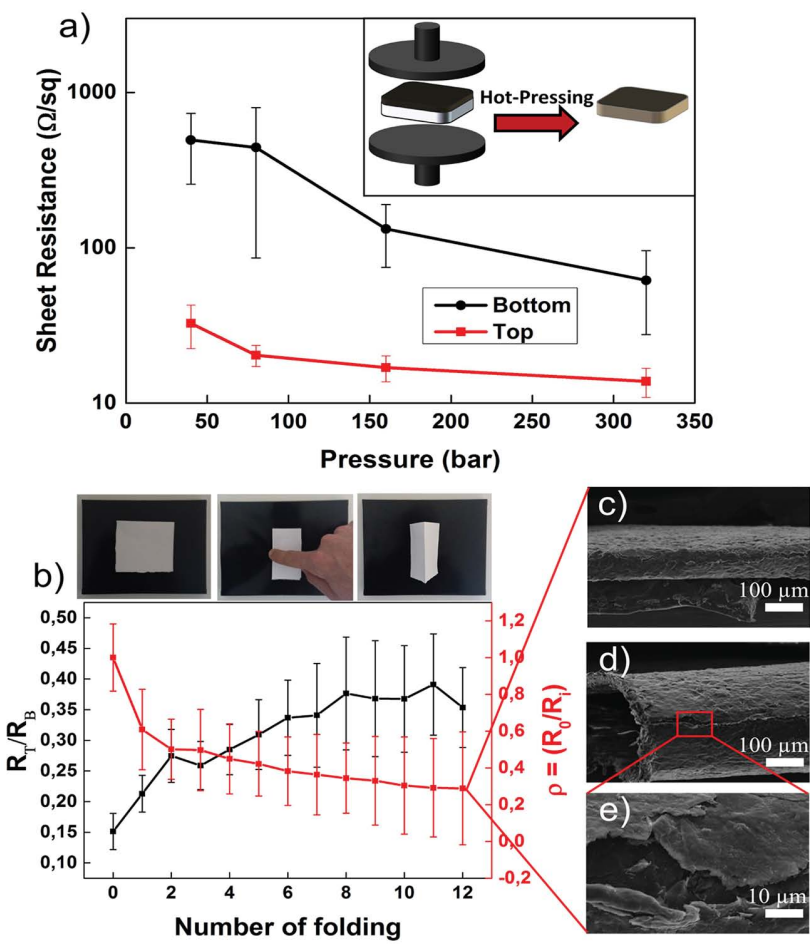

Figure 4. a) Resultant sheet resistance, $R_{\mathrm{s}}$, of top (spray coated) and bottom (untreated) surfaces of the meltblown cellulose sheet as a function of hot press pressure. b) Change in the sheet resistances as a function of folding-unfolding events. Due to folding events, the top surface sheet resistance increases causing the ratio $R_{\mathrm{TO}} / R_{\mathrm{Ti}}$ to decline. $R_{\mathrm{Ti}}$ designates top surface sheet resistance at the $i_{\text {th }}$ folding event and $R_{\mathrm{TO}}$ designates the initial top surface sheet resistance before folding cycles. The ratio $R_{\mathrm{T}} / R_{\mathrm{B}}$ is the sheet resistance ratio between the top (sprayed) and bottom surfaces. The photo above the plot displays folding process. c) SEM image of the folded region after 12-folding cycles. d) SEM image showing a randomly formed crack and e) higher magnification SEM image showing internal structure of the crack.

in the SEM images of Figure S3, Supporting Information, open pores of this cellulose fiber network allow any thermoplastic composite, such as the Mater-Bi-GnP one, to be hot-pressed into its porous network in melted state. Contrary, a similar experiment performed on Xerox paper failed to produce any impregnation due to closed pores as a result of the aforementioned sizing process. The most uniform isotropic conditions are achieved when the meltblown substrate is first spray coated on both sides with the Mater-Bi-GnP coatings followed by a further hot pressing process into the inner texture. In order to maintain the perfect isotropic conditions, sheet resistances of both faces of a one-side sprayed sample with 1:1 polymer-SGnP composition were measured as a function of applied pressure (see inset to Figure 4a). Each measurement is an average of at least six different samples. After impregnation for $1 / 2 \mathrm{~h}$ and cooling down to room temperature, the sheet resistances were measured on both sides of the paper-like conductors. It was found that the downward force corresponding to 320 bars was required and sufficient to minimize the sheet resistance difference between the coated surface and the opposite face. The uncoated surface sheet resistance reached $R_{\mathrm{s}}=80 \Omega \mathrm{sq}^{-1}$ as a result of impregnation at 320 bars and the coated surfaces 
displayed $R_{\mathrm{S}}=15 \Omega \mathrm{sq}^{-1}$. At lower impregnation pressure, at least an order of magnitude difference between the measured resistances on opposite faces was recorded. Therefore, all the samples were impregnated at these conditions.

Afterwards, samples sprayed and hot pressed only on one side were tested against multiple $180^{\circ}$ folding. Note that the folding process was made severe by first pressing and running with fingertip and subsequently placing a $5 \mathrm{~kg}$ weight (a metal cylinder) along the fold line and running it through the folded zone several times (see Figure $4 \mathrm{~b}$ ). The change in sheet resistance due to folding-unfolding events is shown in Figure $4 \mathrm{~b}$. The red line shows change in the normalized resistance, $\rho$, after each press-folding event for the coated top surface. As a result of folding events, the top surface sheet resistance increases determining the enhancement of the ratio $\left(\rho=R_{\mathrm{Ti}} /\right.$ $\left.R_{\mathrm{T} 0}\right)$. In this ratio, $R_{\mathrm{T} 0}$ is the initial sheet resistance and $R_{\mathrm{Ti}}$ designates the sheet resistance at the $i^{\text {th }}$ folding-unfolding event. After 12 press-folding, the absolute sheet resistance increased from $15 \Omega \mathrm{sq}^{-1}$ to $\approx 45 \Omega \mathrm{sq}^{-1}$ by a factor of three. After this point, further press-folding events did not cause any significant increase in $\rho$ even after hundreds of more manual foldingunfolding cycles. It must be mentioned that these initial folding events were conducted by running a weight over the fold line as mentioned earlier. After this process, further (following hundred) folding events were carried out without applying extra weight or pressure over the folded line. Under these conditions, further folding-unfolding events did not determine any significant changes in the electrical conductivity. As a result, these composite conductors should be quite resilient against normal folding events than standard flexible electronic materials. The SEM images reported in Figure $4 \mathrm{c}-\mathrm{e}$ show the state of the surface of the folded zone at the end of 12 folding events. Formation of a crack line is clearly visible on the surface. However, the crack appears to be only superficial not resulting in a complete cracking failure, because the film preserves the conductive paths embedded underneath the crack. Identical measurements on the opposite side, however, indicated that the reduction in the normalized sheet resistance was much less than the coated surface, although the absolute resistance was higher. This was attributed to the fact that due to impregnation from the opposite side the fiber network prevented crack formation and its subsequent propagation in the composite. The ratio between the top and bottom surface sheet resistances $\left(R_{\mathrm{T}} / R_{\mathrm{B}}\right)$ is also plotted in Figure $4 \mathrm{~b}$ (black line). In every fold-unfold measurement cycle, the ratio $R_{\mathrm{B}}>R_{\mathrm{T}}$ remained unchanged. The ratio increased about three times after 12 -fold-unfold events. The increase in the ratio was due to the more significant increase in $R_{\mathrm{T}}$ because of the aforementioned cracks formed at the top surface, while the opposite surface maintained its stability against press-folding due to its embedded structure.

The micromorphology of the spray-coated biopolymer-SGnP layer over the cellulose surface and of the final conductor after hot-press impregnation are shown in the following. In Figure 5a, the as-sprayed surface texture is shown. Randomly oriented $\mathrm{GnP}$ flakes form a rough surface texture just after spray coating. Figure $5 \mathrm{~b}$ shows a higher magnification image in which edges of the upright $\mathrm{GnP}$ flakes are seen. This surface texturing is common in spray-coated polymer-sheet-like nanoparticle slurries. ${ }^{[38]}$ The surface morphology of the paper-like
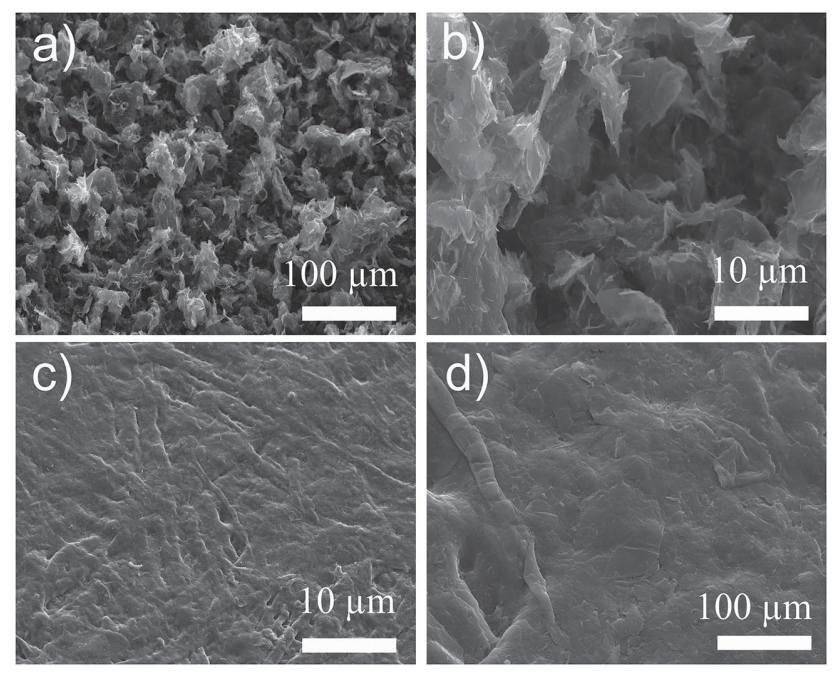

Figure 5. SEM images showing the surface microstructure of the MaterBi-GnP (1:1) paper-like conductor: a) just after spray coating, b) higher magnification showing upright position of the $\mathrm{GnP}$ flakes, c) hot-press impregnation into the fiber network, and d) higher magnification showing traces of cellulose fibers among the impregnated composite.

conductors after hot pressing $\left(175{ }^{\circ} \mathrm{C}\right.$ and 320 bars for $\left.1 / 2 \mathrm{~h}\right)$ demonstrates significant flattening as shown in Figure $5 \mathrm{c}$,d due to the impregnation of the biopolymer-GnP composite into the texture of the cellulose substrate. Note the appearance of microfibers of cellulose within this texture (Figure 5c,d) due to the substrate as a result of the impregnation process.

Further morphological characterization was carried out by TEM measurements on the paper-like foldable conductors. Figure 6a shows that the pores between every adjacent cellulose fiber are filled with the conductive 1:1 biopolymer-SGnP composite. Note that many of the GnP flakes are physically embedded in the cellulose fibers as a result of the impregnation process. This is clearly seen in Figure 5b, where GnP flakes embedded in these cellulose fibers can also form conducting paths through and/or across cellulose fibers.

The embedding process not only induces isotropic electrical conductivity but also enhances the mechanical properties of the cellulose fiber support. Tensile stress-strain properties (average of 10 measurements) of the paper-like conductors (1:1 biopolymer-SGnP) are presented in Figure 7. We also report, for comparison, the measurements on cellulose substrates impregnated only with pure Mater-Bi, i.e., no GnPs. The Young's modulus of the pure cellulose substrate was determined in $\approx 2.2 \mathrm{GPa}$. When the cellulose substrate was impregnated with pure Mater-Bi, the Young modulus of the composite increased to $\approx 3.3 \mathrm{GPa}$, while when it was impregnated with Mater-Bi-SGnP (1:1) composite the Young modulus value reached $\approx 5 \mathrm{GPa}$, more than doubling the value of the initial pure cellulose substrate. On the other hand, paper-like conductors had lower tensile strain compared to cellulose substrates as shown in Figure 7. ${ }^{[39]}$ However, the reduction in strain due to impregnation was not substantial and was within $20 \%-25 \%$. These observations confirm results from previous works ${ }^{[40-42]}$ in which embedding polymers or polymer nanocomposites into natural fiber networks reduced strain due to hindrance of fiber movement and elongation. ${ }^{[40-42]}$ 

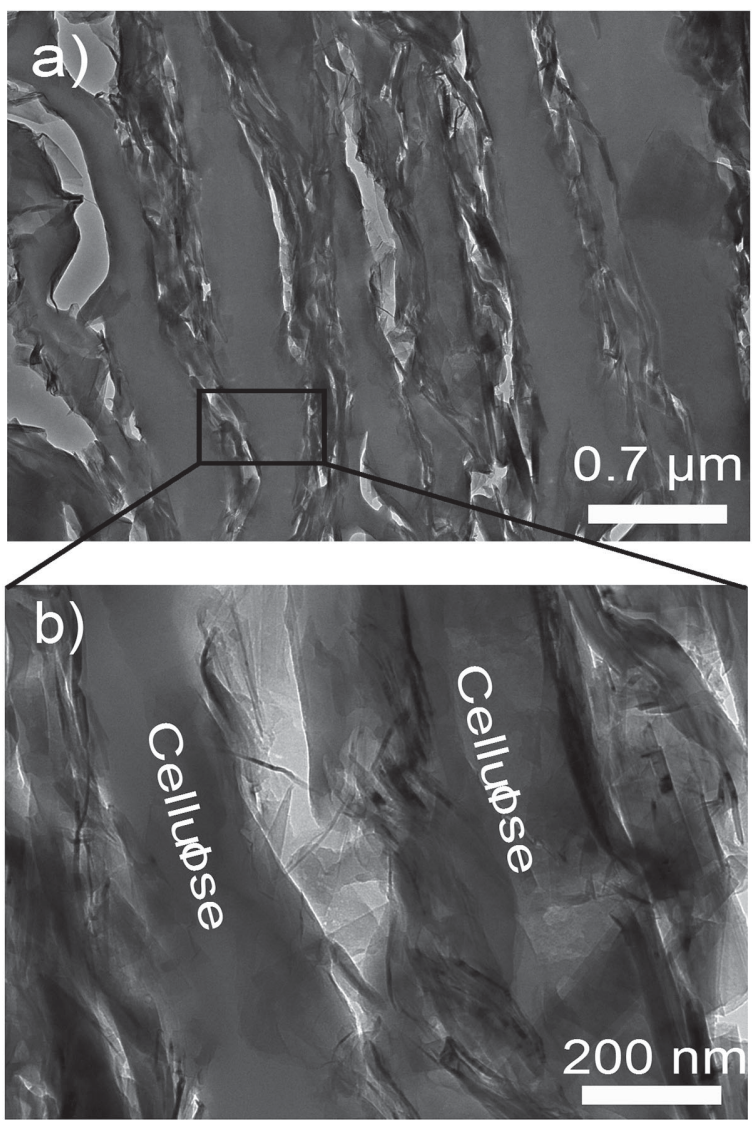

Figure 6. Cross-sectional TEM images of the Mater-Bi-SGnP (1:1) paperlike conductor. a) Lower magnification. The vertical gray lines are cellulose fibers with the interstitial space filled with the Mater-Bi-SGnP composite. b) Higher magnification image. Note that many GnP flakes are embedded into the cellulose fibers.

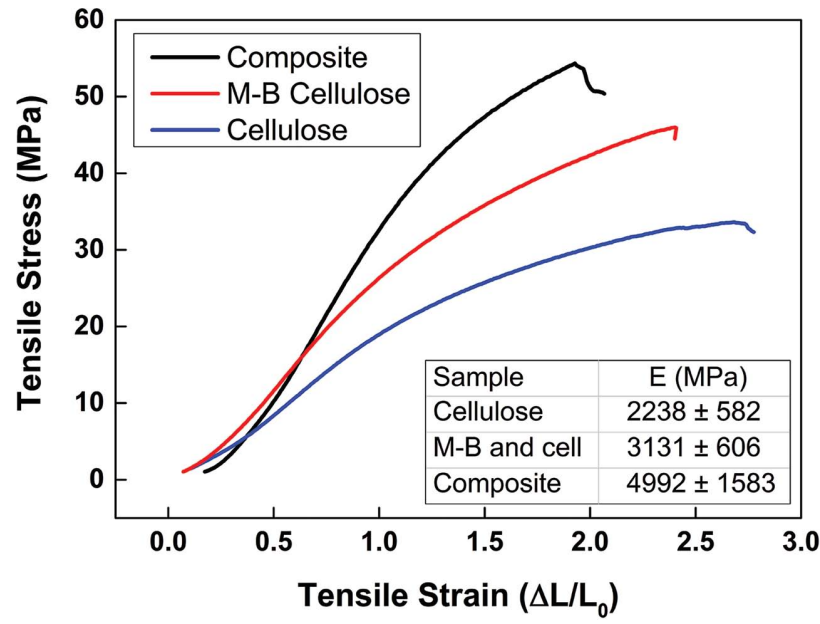

Figure 7. Tensile stress-strain measurements of nonimpregnated cellulose substrate (Cellulose), Mater-Bi impregnated substrate (M-B Cellulose) and Mater-Bi-SGnP (1:1) impregnated (Composite) substrate. Inset displays calculated Young's Moduli.
According to Bledzki and Gassan, ${ }^{[43]}$ Young's modulus of natural cellulose fiber reinforced thermoplastic composites such a polypropylene can range from 2.5 to $7 \mathrm{GPa}$ depending on the type of the surface treatment applied on the cellulose fibers. In many cellulose-thermoplastic composites reported in literature ${ }^{[43]}$ surface chemical treatment of the fibers is generally needed in order to enhance interfacial adhesion between the cellulose-based fibers and the thermoplastic to be impregnated. ${ }^{[43]}$ As shown in Figure 7, the Mater-Bi-graphene composite impregnated into the meltblown cellulose substrates demonstrate Young's modulus close to $5 \mathrm{GPa}$. This value is achieved without the need of any chemical surface treatment of the cellulose fibers, a great advantage because it simplifies the fabrication process reducing the costs.

None of the as-prepared composites were hydrophobic. The original cellulose substrate absorbs water completely and no contact angle can be measured. The wetting characteristics of pure Mater-Bi were reported in a previous work. ${ }^{[4]}$ Mater-Bi impregnated cellulose sheets display contact angles close to $75^{\circ}$. When SGnP or DGnP are blended into the matrix, no significant changes in the wettability of the composites occurred and static contact angles were close to $70^{\circ}$. Hence, the composite conductors retain resistance against wetting by water. When only plain GnP flakes were sprayed on the cellulose substrates the particles could be removed easily by rubbing. After impregnation by hot pressing, GnP flakes could be removed from the cellulose by simple tape peel action or by continuous rubbing. However, since the conductors contain Mater-Bi as matrix for GnPs, the polymer acted as a binder between the cellulose and the GnP flakes. A series of tape peel tests has shown that the composites maintain good adhesion to the cellulose texture with adhesion strengths close to $0.44 \mathrm{~N} \mathrm{~mm}^{-1}$. Further work will focus on rendering these composites completely waterproof and self-cleaning.

\section{Conclusion}

In summary, we have shown a simple, fully reproducible method to embed a biopolymer-GnP composite into a cellulose fiber network forming foldable isotropic paper-like conductors. The resultant materials exhibit exceptional electrical properties (a sheet resistance of $10 \Omega \mathrm{sq}^{-1}$ ) and excellent folding stability. Electrical percolation occurs at $6 \mathrm{wt} \%$ (or at a volume fraction approximately of 0.025 ). These conductors can be easily distinguished from ink-jet printed conductive Xerox papers in the sense that impregnation enables formation of an isotropic paper-like conductor. Many potential application areas can be envisioned including environmentally friendly disposable flexible electronics, bio-based wearable conductors and electromagnetic shielding applications, just to name a few.

\section{Experimental Section}

Materials and Methods: SGnP with thickness of 6-8 nm and lateral size of $5 \mu \mathrm{m}$ were purchased from Strem Chemicals. DGnP were donated by Directa Plus (grade Ultra G+, 7-9 nm thick, and $\approx 20 \mu \mathrm{m}$ in lateral size). Colloidal graphite suspension ( $\mathrm{C} 126$; isopropanol base) was purchased from TAAB Laboratories Equipment Ltd, UK. This 
suspension was precipitated and dried and then the graphitic flakes were redispersed in chloroform. Mater-Bi bioplastic (license number 14) was obtained from Novamont S.p.A, Italy. Chloroform was acquired from Sigma-Aldrich. Cellulose substrates ( $30 \mu \mathrm{m}$ thick) were purchased from Korff (Art. Num. 60285). Teflon anti-attachment films, used during the hot-pressing process, were obtained from Advent Research Material (Art. Num. FP823338). They were used to prevent possible sticking of the conductors on the surfaces of the press.

Mainly, a $50 \mathrm{wt} \%$ dispersion of Mater-Bi $(0.35 \mathrm{~g})$ and SGnP or DGnP $(0.35 \mathrm{~g})$ was prepared in chloroform $(30 \mathrm{~mL})$ to ensure good dispersion. ${ }^{[36]}$ The dispersion was then probe sonicated $(750 \mathrm{~W}, 40 \%$ amplitude, $20 \mathrm{kHz}$, four times for $15 \mathrm{~s}$ ) using a Sonics \& Materials, Inc., (Model Num. VCX750) and sprayed (18 cm, 2.2 bar) on the substrates using a Paasche air brusher (VL siphon feed, $0.73 \mathrm{~mm}$ nozzle). After drying, the unpolished paper-like conductor was cut in rectangles $(2.5 \times 5 \mathrm{~cm})$ and hot-pressed (from 40 to 320 bar, $30 \mathrm{~min}, 175{ }^{\circ} \mathrm{C}$ ) using a Specac-Atlas Power Presses T8 or a Specac-Atlas 15T Manual Hydraulic press in order to impregnate the sprayed composite into the fiber network. Also in this case, it was possible to spray both sides of the paper so that isotropic conductors could be realized. In order to obtain the cross-sectional TEM images, first, samples were cut with a steel blade in small triangular-shaped stripes and then dehydrated with ethanol (99.9\%) for $8 \mathrm{~h}$, changing the solvent every $2 \mathrm{~h}$. Subsequently, the samples were embedded in an epoxy resin. A low viscosity Spurr resin (SPI-Chem), having a long curing time, was chosen in order to minimize structural deformation of the samples during epoxy encapsulation. Once the resin has hardened, $180 \mathrm{~nm}$ thick sections were cut with a Leica EMU C6 ultramicrotome.

Measurements: Raman spectra of Mater-Bi and the composite with GnPs were performed with a Raman spectrometer (Horiba HR800 UV) with an excitation wavelength of $632.8 \mathrm{~nm}$. FTIR spectra were collected using a Bruker Vertex 70v to chemically characterize Mater-Bi and cellulose. A four-probe resistance analyzer (Signatone 1160 probe station) was used to measure the sheet resistance. Before electrical measurements, the as-prepared sample surfaces were gently wiped with polishing tissues (Kimtech Science Precision Wipes -Art. Num. 05511) and air blasted (1 bar) to remove any dust and contaminants. Silver paste (SPI Conductive Silver Paint, resistivity $\approx 0.01 \Omega \mathrm{sq}^{-1}$ ) electrodes were painted on the conductive surfaces, keeping apart $2 \mathrm{~mm}$ spacing. To determine the Young's Modulus, a tensile tester (Instron 3365) was used; samples were stretched by applying a strain rate of $1 \mathrm{~mm} \mathrm{~min}$. Thermogravimetric analysis was conducted using a TA instruments apparatus (model Q500). The surface morphology was studied with a JEOL microscope (model JSM-6490LA) operating at an acceleration voltage of $15 \mathrm{kV}$. TEM images were collected with a JEOL JEM 1011 electron microscope, operating at an acceleration voltage of $100 \mathrm{kV}$, with a $11 \mathrm{Mp}$ fiber optical charge-coupled device (CCD) camera (Gatan Orius SC-1000). A micrometer from Mitutoyo (series 293) was utilized to determine thicknesses of samples and cellulose substrates. The LED chips were made from Cree lights (model CLP6, white, $4.4 \mathrm{~V}, 32000 \mathrm{mlm}$ ). They were assembled on copper plates to resemble the logo of IIT.

SGnP DLS measurements were carried out with a Malvern Zetasizer Nano ZS, using a $632.8 \mathrm{~nm} \mathrm{He} / \mathrm{Ne}$ laser. Dilute chloroform dispersions of the SCnP flakes were kept in quartz cuvettes with $10 \mathrm{~mm}$ path length. The measurements were conducted in backscatter mode at an angle of $173^{\circ}$. Samples were equilibrated to $25^{\circ} \mathrm{C}$ for 2 min prior to measurement. Values for solvent (chloroform) viscosity at $25^{\circ} \mathrm{C}$, as provided by the solvent supplier, were entered into the software. A default measurement duration setting was used, with automatic measurement positioning and attenuation. Three different samples were analyzed in order to ensure repeatability of the analysis. AFM measurements were performed with a SPI 4000 system in tapping mode. The samples were deposited from chloroform suspensions (SGnP concentration $\approx 0.5 \%$ by weight) onto freshly cleaved mica substrates, dried by evaporation. The measurements were performed in air at ambient temperature and pressure. The adhesion strength of the graphene-biopolymer composites was tested by manually peeling high tack $3 \mathrm{M}$ tape $(3 \mathrm{M}, \mathrm{CN} 4190$ Polyester tape with acrylic adhesive, Adhesion to steel is $4.4 \mathrm{~N} / 10 \mathrm{~mm}$ ) applied on the conductor surfaces. Peeled tapes removed only a very small fraction of the coating. Over the zones where the tape was peeled away, electrical conductivity remained unchanged and was not lost after repeated tapepeeling tests. This outcome provides empirical confirmation of the durability of the paper-like conductors.

\section{Supporting Information}

Supporting Information is available from the Wiley Online Library or from the author.

\section{Acknowledgements}

The authors gratefully acknowledge funding from the European Union (EU) under Graphene Flagship (Contract 604391). The authors also acknowledge continuous support from Drs. Massimiliano Bianchi and Laura Rizzi of DIRECTA PLUS S.p.A. The authors also acknowledge experimental assistance from UIC Research Resources Center, Chicago, USA.

Received: July 13, 2015

Revised: September 8, 2015

Published online: October 26, 2015

[1] Y.-L. Tai, Z.-G. Yang, J. Mater. Chem. 2011, 21, 5938.

[2] Z. Ling, C. E. Ren, M.-Q. Zhao, J. Yang, J. M. Qiu, J. Giammarco, M. W. Barsoum, Y. Gogotsi, Proc. Natl. Acad. Sci. USA 2014, 111, 16676

[3] D. Tobjörk, R. Österbacka, Adv. Mater. 2011, 23, 1935.

[4] A. Russo, B. Y. Ahn, J. J. Adams, E. B. Duos, J. T. Bernhard, J. A. Lewis, Adv. Mater. 2011, 23, 3426.

[5] L. Hu, G. Zheng, J. Yao, N. Liu, B. Weil, M. Eskilsson, E. Karabulut, Z. Ruan, S. Fan, J. T. Bloking, M. D. McGehee, L. Wagberg, Y. Cui, Energy Environ. Sci. 2013, 6, 513.

[6] W. Shen, Y. Filonanko, Y. Truong, I. H. Parker, N. Brack, P. Pigram, J. Liesegang, Colloids Surf., A 2000, 173, 117.

[7] S. Kalia, B. S. Kaith, I. Kaur, Polym. Eng. Sci. 2009, 49, 1253.

[8] X. Li, J. Tian, W. Shen, Cellulose 2010, 17, 649.

[9] I. M. T. Moutinho, P. J. T. Ferreira, M. L. Figueiredo, Bioresources 2011, 6, 4259

[10] A. Ferrari, F. Bonaccorso, V. Falko, K. S. Novoselov, S. Roche, P. Bøggild, S. Borini, F. H. L. Koppens, V. Palermo, N. Pugno, J. A. Garrido, R. Sordan, A. Bianco, L. Ballerini, M. Prato, E. Lidorikis, J. Kivioja, C. Marinelli, T. Ryhanen, A. Morpurgo, J. N. Coleman, V. Nicolosi, L. Colombo, A. Fert, M. Garcia-Hernandez, A. Bachtold, G. F. Schneider, F. Guinea, C. Dekker, M. Barbone, Z. Sun, C. Galiotis, A. N. Grigorenko, G. Konstantatos, A. Kis, M. Katsnelson, L. Vandersypen, A. Loiseau, V. Morandi, D. Neumaier, E. Treossi, V. Pellegrini, M. Polini, A. Tredicucci, G. M. Williams, B. H. Hong, J.-H. Ahn, J. M. Kim, H. Zirath, B. J. van Wees, H. van der Zant, L. Occhipinti, A. Di Matteo, I. A. Kinloch, T. Seyller, E. Quesnel, X. Feng, K. Teo, N. Rupesinghe, P. Hakonen, S. R. T. Neil, Q. Tannock, T. Lofwander, J. Kinaret, Nanoscale 2015, 7, 4598.

[11] Y. Zhu, S. Murali, W. Cai, X. Li, J. W. Suk, J. R. Potts, R. S. Ruoff. Adv. Mater. 2010, 22, 3906.

[12] J. Hassoun, F. Bonaccorso, M. Agostini, M. Angelucci, M. G. Betti, R. Cingolani, M. Gemmi, C. Mariani, S. Panero, V. Pellegrini, B. Scrosati, Nano Lett. 2014, 14, 4901.

[13] F. Bonaccorso, L. Colombo, G. Yu, M. Stoller, V. Tozzini, A. Ferrari, V. Pellegrini, Science 2015, 347, 1246501. 
[14] G. Fiori, F. Bonaccorso, G. Iannaccone, T. Palacios, D. Neumaier, A. Seabaugh, S. K. Banerjee, L. Colombo, Nat. Nanotechnol. 2014, 9, 768.

[15] M. Terrones, O. Martín, M. González, J. Pozuelo, B. Serrano, J. C. Cabanelas, S. M. Vega-Díaz, J. Baselga, Adv. Mater. 2011, 23, 5302.

[16] T. Kuilla, S. Bhadra, D. Yao, N. H. Kim, S. Bose, J. H. Lee, Prog. Polym. Sci. 2010, 35, 1350.

[17] A. Bianco, H.-M. Cheng, T. Enoki, Y. Gogotsi, R. H. Hurt, N. Koratkar, T. Kyotani, M. Monthioux, C. R. Park, J. M. D. Tascon, J. Zhang, Carbon 2013, 65, 1.

[18] L. Kavan, J. H. Yum, M. Grätzel, ACS Nano 2011, 5, 165.

[19] H.-P. Fink, J. Ganster, A. Lehmann, Cellulose 2014, 21, 31.

[20] C. Bastioli, Polym. Degrad. Stability 1998, 59, 263.

[21] D. Puglia, A. Tomassucci, J. M. Kenny, Polym. Adv. Technol. 2003, 14, 749.

[22] I. S. Bayer, V. Caramia, D. Fragouli, F. Spano, R. Cingolani, A. Athanassiou, J. Mater. Chem 2012, 22, 2057.

[23] T. J. Mason, Sonochemistry, Oxford University Press, New York 1999, Ch. 1.

[24] A. Capasso, A. E. Del Rio Castillo, H. Sun, A. Ansaldo, V. Pellegrini, F. Bonaccorso, Solid State Commun. 2015, DOI:10.1016/j. ssc.2015.08.011.

[25] Y. Li, J. Zhu, S. Wei, J. Ryu, L. Sun, Z. Guo, Macromol. Chem. Phys 2011, 212, 1951

[26] J. Hicks, A. Behnam, A. Ural, Appl. Phys. Lett. 2009, 95, 213103.

[27] K. Jost, G. Dion, Y. Gogotsi, J. Mater. Chem. A 2014, 2, 10776.
[28] S. Stankovich, D. A. Dikin, G. H. B. Dommett, K. M. Kohlhaas, E. J. Zimney, E. A. Stach, R. D. Piner, S. T. Nguyen, R. S. Ruoff, Nature 2006, 442, 282

[29] T. Kuilla, S. Bhadra, D. Yao, N. H. Kim, S. Bose, J. H. Lee, Prog. Polym. Sci. 2010, 35, 1350

[30] J. R. Potts, D. R. Dreyer, C. W. Bielawski, R. S. Ruoff, Polymer 2011, $52,5$.

[31] W. Bauhofer, J. Z. Kovacs, Comp. Sci. Technol. 2009, 69, 1486.

[32] A. Celzard, J. F. Marêché, Physica A 2003, 317, 305.

[33] S. De, J. N. Coleman, ACS Nano 2010, 4, 2713.

[34] L. He, S. C. Tjong, Nanoscale Res. Lett. 2013, 8, 132.

[35] M. Yoonessi, J. R. Gaier, ACS Nano 2010, 4, 7211.

[36] Y. Zhan, M. Lavorgna, G. Buonocore, H. Xia, J. Mater. Chem. 2012, 22, 10464.

[37] M. B. Heaney, Phys. Rev. B 1995, 52, 12477.

[38] A. Steele, I. Bayer, E. Loth, Nano Lett. 2009, 9, 501.

[39] M. A. Rafiee, J. Rafiee, Z. Wang, H. Song, Z.-Z. Yu, N. Koratkar, ACS Nano 2009, 3, 3884.

[40] H. Kim, A. A. Abdala, C. W. Macosko, Macromolecules 2010, 43 6515.

[41] D. Han, L. Yan, W. Chen, W. Li, P. R. Bangal, Carbohydr. Polym. 2011, 83,966

[42] M. Monti, M. Rallini, D. Puglia, L. Peponi, L. Torre, J. M. Kenny, Comp., Part A. 2013, 46, 166.

[43] A. K. Bledzki, J. Gassan, Prog. Polym. Sci. 1999, 24, 221.

[44] A. Milionis, R. Ruffilli, I. S. Bayer, RSC Adv. 2014, 4, 34395. 